\title{
The appeal of the Functional Fitness MOT to older adults and health professionals in an outpatient setting: a mixed-method feasibility study
}

This article was published in the following Dove Press journal: Clinical Interventions in Aging

\author{
Lex $D$ de Jong ${ }^{1,2}$ \\ Andy D Peters ${ }^{3}$ \\ Sheena Gawler ${ }^{4,5}$ \\ Nina Chalmers ${ }^{6}$ \\ Claire Henderson ${ }^{6}$ \\ Julie Hooper ${ }^{6}$ \\ Robert Laventure ${ }^{5,7}$ \\ Laura McLean ${ }^{6}$ \\ Dawn A Skelton ${ }^{1,5}$
}

'Centre for Living, School of Health and Life Sciences, Glasgow Caledonian University, Glasgow, UK; ${ }^{2}$ School of Physiotherapy and Exercise Science, Faculty of Health Sciences, Curtin University, Perth, WA, Australia;

${ }^{3}$ Delivering Better Care Hub, Western General Hospital, Edinburgh, UK;

${ }^{4}$ Research Department of Primary Care and Population Health, University College London, London, UK; ${ }^{5}$ Later Life Training Ltd, Killin, UK; ${ }^{6}$ Edinburgh Community Physiotherapy Service, Edinburgh, UK; ${ }^{7}$ British Heart Foundation National Centre for Physical Activity and Health, Loughborough, UK
Correspondence: Dawn A Skelton Centre for Living, School of Health and Life Sciences, Glasgow Caledonian University, A I OIg Govan Mbeki Building, Cowcaddens Road, Glasgow, G4 OBA, UK Tel +44 I4I 33। 8792

Email dawn.skelton@gcu.ac.uk
Purpose: To understand the views and perceptions regarding the Functional Fitness MOT (FFMOT), a battery of functional tests followed by a brief motivational interview, of both the older people undergoing it and the health professionals delivering it.

Patients and methods: Physically inactive older adults $(n=29)$ underwent the FFMOT and subsequently attended focus groups to share their perceptions of it and to discuss the barriers, motivators, health behavior change, and scope to improve physical activity (PA) levels. PA levels were recorded at baseline and again at 12 weeks together with a post-intervention questionnaire concerning behavior change. Participating physiotherapists and technical instructors were interviewed.

Results: Most participants felt they had learned about their abilities and comparisons with their peers, had a change in perception about the importance of good balance and strength, and felt the FFMOT helped raise their awareness of local and self-directed physical activity opportunities. Most felt their awareness of the need for PA had not changed, but 25\% of participants started a new organized PA opportunity. The health professionals perceived the FFMOT as being easy to administer, educating, and motivating for participants to increase their PA. Space, time, finances, and insecurity about having the necessary skills to conduct the FFMOTs were seen as barriers in implementing the FFMOT in daily practice.

Conclusion: Over half of those offered the FFMOT accepted it, suggesting it is appealing. However, most participants felt they were already active enough and that their awareness of the need for PA had not changed. There were positive perceptions of the FFMOT from both professionals and older people, but both felt the FFMOT could be held in a community venue. The overall findings suggest that the FFMOT is feasible in the clinical setting, but its effectiveness has yet to be determined.

Keywords: aged, health behavior, health services for the aged, physical activity, physical fitness, physical therapists

\section{Introduction}

Increasing physical activity (PA) can bring substantial health benefits, ${ }^{1}$ improved health care outcomes, and reduced health care $\operatorname{costs}^{2}$ for older adults. PA guidelines recommend that older adults engage in activities which improve strength and balance at least twice a week, avoid prolonged seated periods, and undertake moderateto-vigorous intensity PA (MVPA) for at least 150 minutes per week in bouts of 10 minutes. $^{2}$ However, few older adults meet the current PA recommendations..$^{3-5}$ 
In the UK, only one in five people aged 65-74 years and one in ten over 75 years achieve the recommended activity levels. ${ }^{6}$ Those meeting the activity guidelines for strength and balance activities ${ }^{7}$ are even lower. The prevalence of sedentary behavior is particularly prevalent in older populations, with an average of 9.4 hours sitting a day. ${ }^{8}$ Effective approaches to encourage increased PA in older adults are still needed. ${ }^{9}$

Recently, one study showed that older people recruited through general practice and undergoing a 6-month group exercise intervention had increased their self-reported moderately vigorous PA by about 15 minutes a day, a year after the intervention finished. ${ }^{10}$ However, this weekly group-based approach was more expensive than home-based exercises. Also, only $13 \%$ of eligible participants expressed an interest in participating in these exercise classes, which suggests that a service based on group exercises may be unappealing to many older people. One solution to these limitations might be to use a cost-effective and brief intervention that is more tailored to the individual in terms of suggesting multiple local or self-directed activities for the person to choose from to increase the chance of uptake.

The Functional Fitness MOT (FFMOT) is a theory-driven intervention, based on the Capability, Opportunity, Motivation, and Behavior model. ${ }^{11}$ This health behavior change framework, which can be applied to people's behavior necessary to increase their PA, conceptualizes that one requires the capability (knowledge, awareness, self-efficacy), opportunity (environmental, social), and motivation to change behavior (Figure 1).

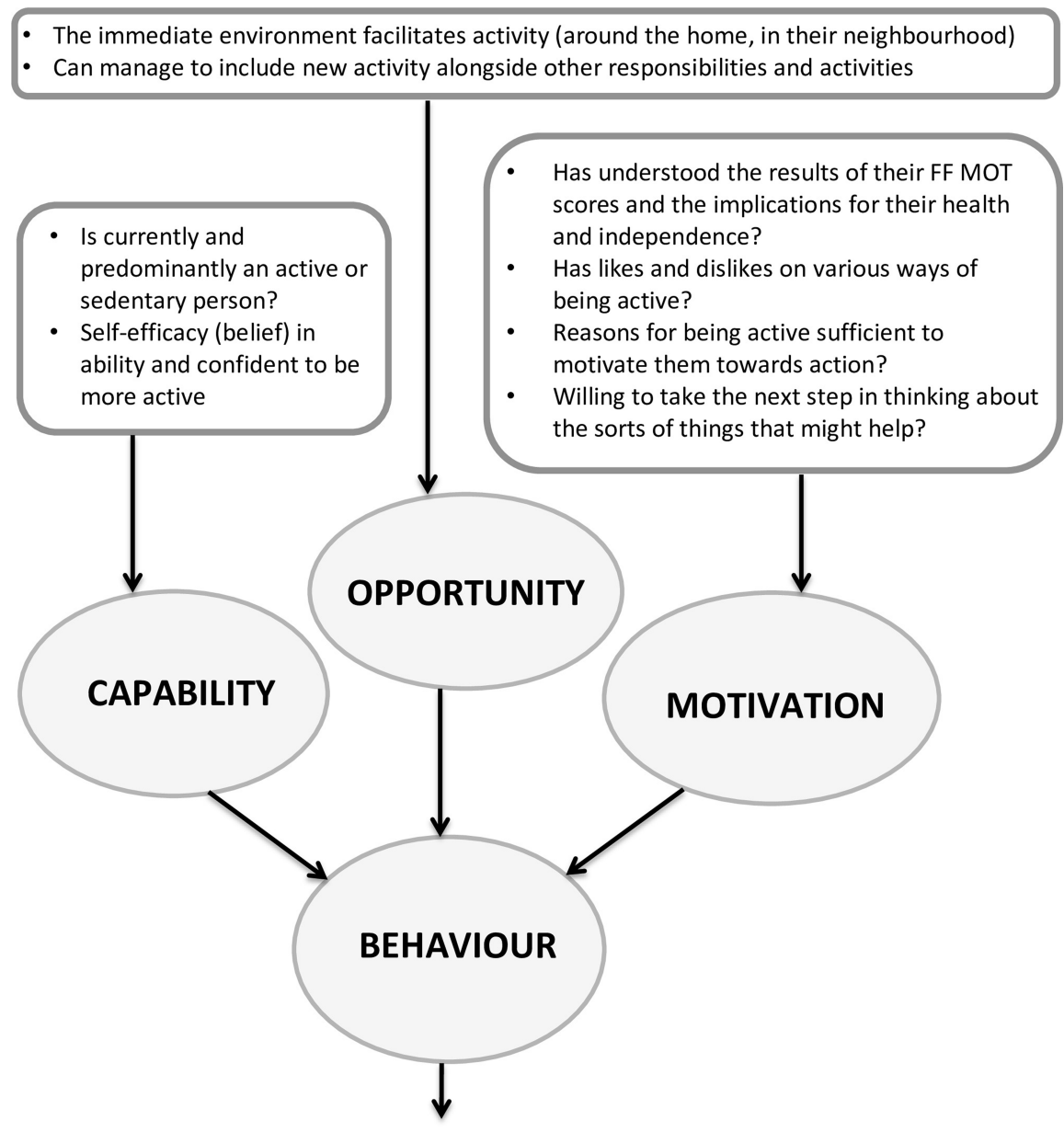

The COM-B model suggests that participants need be confident and understand all three areas in order to be successful in making such a change to their behaviour.

Figure I The Functional Fitness MOT (FFMOT) is a theory-driven intervention, based on the Capability, Opportunity, Motivation, and Behavior (COM-B) model." Notes: Reproduced with permission from Later Life Training; Glasgow Caledonian University. The Functional Fitness MOT: Course Manual and Resources. Edition I. Killin: Later Life Training; 2017. Copyright Later Life Training 2017..$^{33}$

Abbreviation: FFMOT, Functional Fitness MOT. 
The FFMOT was mainly developed as a means to raise knowledge and awareness of the PA guidelines, particularly the newer strength and balance guidelines and the recommendation to sit less. ${ }^{12}$ The personalized conversation during and after the functional tests aims to provide a motivational conversation with older people about PA in order to increase intention to sit less and to do more strength and balance activities and moderate PA. It starts with a battery of validated performance tests, which provides a means of assessing key physiological parameters that are associated with functional mobility in independent older adults aged between 60 and 90 years. The idea for this test battery was derived from a previous study by Rikli and Jones. ${ }^{13}$ The FFMOT procedure involves one face-to-face session during which seven ageappropriate functional fitness tests are administered, followed by immediate feedback on the person's abilities in each of these tests in relation to normal values for their age and sex. Some of these tests are actually used in strength and balance training programs (eg, chair rise and one leg stand). They have no "floor" effects which means they are achievable even in those not currently active and can show the person's "capability" in achieving these tasks. Subsequently, tailored advice is given on how to improve the components of fitness in which they are below "normal". Local activity opportunities (indoors and outdoors), ideas for activities they can do at home for free, and the benefits of being active in a group (social and group cohesion) are all discussed to address preferences and environment. This brief motivational set of tests and discussion is based on the principles of health behavior change and incorporates awareness raising, breaking down barriers, improving self-efficacy, building on history of activity, incorporating needs and preferences, and goal setting. ${ }^{14}$ The older adult receives a personal copy of their assessments and action plan to take away and reflect on. The ultimate goal of the FFMOT is to engage older people in health behavior change discussions and to direct them to appropriate local activity resources. Although there has been a report as to its utility from several professionals trained to deliver the FFMOT to older people, ${ }^{12}$ there has been no research on the use of the FFMOT as a brief intervention.

The aims of this study were to assess whether the FFMOT, provided in a health care setting, was appealing to older patients of a community physiotherapy service and to understand the views and perceptions of the older people undergoing the FFMOT regarding the intervention, as well as the views of the physiotherapy staff delivering the intervention. Secondary aims were to assess the feasibility of carrying out a Phase II pilot randomized controlled trial
(RCT) of the FFMOT, in the context of a community physiotherapy service, by establishing whether enough patients could be recruited and retained in the study and whether enough outcome data could be generated.

\section{Patients and methods Design}

The study protocol with the key aims and design of this mixed-method Phase I feasibility study have been reported in more detail previously. ${ }^{15}$ In brief, this design is appropriate to evaluate a new intervention like the FFMOT before it is introduced in a clinical setting ${ }^{16}$ and can be used to evaluate whether it is acceptable and practical for the participants as well as the staff delivering it. This design was also chosen to evaluate the feasibility of carrying out a Phase II pilot RCT of the FFMOT in the future by establishing whether enough patients can be recruited and retained in the study and whether enough relevant outcome data can be generated.

\section{Ethical and organizational review}

Ethics approval was granted by the National Health Service (NHS) South East Scotland Research Ethics Committee 01, Scotland UK (Reference 15/SS/0118) on July 20, 2015. Approval was also granted by the NHS Lothian Research \& Development Office (Reference 2015/0283) on August 3, 2015. The study was sponsored by Glasgow Caledonian University (Reference RIE13-127). Participant recruitment started on October 5, 2015. As initially it was unclear to the research team that a mixed-method Phase I feasibility study fitted the International Committee of Medical Journal Editors definition of a clinical trial, the trial was registered retrospectively on February 2, 2016 (ISRCTN38950042; http://www.isrctn. com/ISRCTN38950042). The protocol paper ${ }^{15}$ of the trial was accepted on April 4, 2016. When all 12-week follow-up data were received (June 1, 2016), the trial was completed.

\section{Sample size}

As this was a feasibility study, no formal power calculation was carried out. The aim was to recruit 30 participants and retain 21 at 12-week follow-up following a conservative estimate of $70 \%$ retention.

\section{Participants}

A convenience sample of outpatients with musculoskeletal (MSK) conditions was identified and recruited from among the caseloads of community physiotherapists (PTs) working in two physiotherapy clinics from one UK health board area. The inclusion criteria were as follows: 1 ) age $\geq 60$ years, 
2) not physically active for at least 30 minutes on 5 days or more, or for at least 150 minutes in total in the past week as indicated by the questions on the Scottish Physical Activity Screening Question (Scot-PASQ), ${ }^{17}$ and 3) those who were interested in increasing their level of PA where this was seen as an appropriate goal by the screening PT. Participants were excluded if the screening PT identified the health risks (contraindications to exercise, eg, cardiovascular disease), and if they had been diagnosed with moderate/severe cognitive impairment, a learning disability, severe mental illness, or if the screening PT believed that any of these impairments/ disorders was present. Participants continued to receive standard physiotherapy assessment and intervention as deemed appropriate by the screening PT, irrespective of their involvement in the study. Written informed consent was obtained from all participants.

\section{Functional fitness MOT}

Each recruited participant was provided with an individual FFMOT session at one of the two recruitment clinics, which lasted for 45-60 minutes. Two trained technical instructors (TIs) each delivered 16 and 13 FFMOTs, respectively, over seven morning sessions. The supervising PTs provided oversight of the TIs and the embedding of the FFMOT into the service, but were not with the participants during the FFMOTs. The FFMOT in this study comprised six (of the seven original) standardized, validated, age-appropriate tests: the 30-second chair stand, the chair sit and reach, the back scratch, the 8-foot up and go, the handgrip strength, and the single-leg stance. ${ }^{18,19}$ The one FFMOT test not used in this setting, because of space and time issues, was the 6-minute walk test. The results of the six tests were used by the TIs to discuss different components of fitness with the participants, to highlight the individuals' strengths and weaknesses in these components, and to provide them with personal feedback on performance in comparison to sex- and age-referenced normative values. Subsequently, a discussion around the person's activity history, needs and preferences took place so that information about local opportunities to engage in PA could be introduced and encouraged. Specific goal setting (short and longer term) was discussed, and each participant was provided with information about appropriate local activity opportunities, advice to sit less or break up any prolonged periods of sitting, and home strength and balance exercises, based on their FFMOT results. This information was provided in the form of written booklets/leaflets, which contained links to the websites of some of the opportunities available. For those who wished to exercise at home, home exercise booklets were provided. ${ }^{20}$

\section{Training in delivery of the FFMOT}

Prior to the trial, both TIs received training in the delivery of the FFMOT and in motivation and support strategies for engaging older adults in PA..$^{21}$ The TIs were not involved in the design of the study or the routine treatment of the participants.

\section{Data collection Quantitative data}

Data collected from patients included the clinic at which the patient was assessed/treated, sex, age, postcode, responses to the three questions in the Scot-PASQ, ${ }^{17}$ eligibility criteria, whether the study was introduced verbally to the patient, whether the patient accepted the study information pack, and whether the patient completed and returned a contact details form to the research team. The Community Healthy Activities Model Program for Seniors (CHAMPS) physical activity questionnaire ${ }^{22}$ was administered a few minutes before the FFMOT and (returned by post) at follow-up 12 weeks later to measure the levels and types of PA. This questionnaire asks participants to recall the frequency and duration (hours) of 41 different social, leisure, household, and physical activities that they have done in a typical week within the last 4 weeks. The CHAMPS is valid and reliable in this age group. ${ }^{23}$ Twelve weeks after the FFMOT session a bespoke post-intervention questionnaire was also completed (returned by post). This questionnaire measured the participants' contact with PA-focused community organizations and facilities since undergoing the FFMOT.

\section{Qualitative data}

Three focus groups were organized, so that the FFMOT participants could share their perceptions of the intervention with the researchers. Focus group dates allowed most participants to provide their views between 2 and 8 weeks after their initial FFMOT. Each focus group took place at the same clinic where the FFMOT had taken place and was moderated by a male researcher (LDdJ). The discussion guide contained a set of semi-structured, open-ended questions asking participants about their reason(s) for taking part in the study, their PA awareness, the appeal of the FFMOT, and their experiences of the study procedures. ${ }^{15}$ Specific questions relating to the participants' PA awareness (reasons for not adhering to PA guidelines, perception changes after the FFMOT, views on and awareness about the importance and benefits of PA, perceived importance of and changes in PA behavior since the FFMOT, and changes in awareness of local opportunities to become more physically active) were based on the constructs of the COM-B health behavior change framework. ${ }^{11}$ The participants' experiences of the 
study procedures are not presented here, but will be used by the researchers in a definitive trial to ensure participation is maximized. Attendance at the FFMOT and focus group, completion of pre-assessment CHAMPS questionnaire, and return of completed follow-up questionnaires among consented participants were also monitored and recorded to calculate recruitment and retention rates.

Six semi-structured individual interviews of up to 60 minutes, with the four supervising PTs and the two TIs, were held within 2 weeks of the final FFMOT sessions. Four of the interviews took place in a private room at the physiotherapy clinic where the FFMOTs took place. Three of these were moderated by two researchers (LDdJ, SG), and one by only one (LDdJ). Two PTs were interviewed by telephone (LDdJ). All staff were interviewed about their previous experiences with, and background knowledge of, working with older people, therapy, fitness tests, exercise, research, and their perceptions of the FFMOT, and the FFMOT in the context of a community physiotherapy service.

\section{Data analysis Quantitative data}

Reasons for exclusion, participant recruitment and retention rates, number of completed questionnaires returned, and incidence of missing questionnaire item responses were explored using descriptive statistics. Descriptive statistics were also used to explore demographic differences (including sex, age, the clinic where screening occurred, the Scottish Index of Multiple Deprivation ${ }^{24}$ rank of participant's home postcode, and traveling distance from home to clinic [calculated using an online tool ${ }^{25}$ between 1) eligible and non-eligible patients among those screened, 2) eligible patients who accepted an information pack and those who did not, 3) eligible patients who were recruited and those who were not, and 4) completers and non-completers]). Screening data for non-consented patients were anonymized, stored, and analyzed securely and wholly within the NHS health care team, rather than by the university researchers who processed the data relating to consented participants. The anonymized quantitative data can be found at https://edshare.gcu.ac.uk/3386/. CHAMPS data were compared between baseline and 12-week follow-up to provide a provisional estimate of effect size to inform the design of a future trial. All quantitative analyses were performed using SPSS Statistics v24.

\section{Qualitative data}

All focus group discussions and individual interviews were recorded onto an encrypted digital recorder. All recordings were fully transcribed verbatim and anonymized. Transcripts were not returned to participants for comments or corrections. Qualitative content analysis was performed, a method for analyzing communication in different steps in a systematic manner ${ }^{26}$ and a process of interpretation that focuses on similarities and differences and results in the organization of data into categories and themes. ${ }^{27}$ Analysis was carried out by three of the authors (LDdJ, SG, DAS). PTs and TIs provided feedback on the findings from the thematic analysis, and all authors agreed on the final themes. Member checking with the older adults in the study was not performed.

\section{Results}

Two hundred and ninety-nine patients were screened for eligibility. The flow of participants through each stage of the trial, from initial screening to 12 -week follow-up, is presented in Figure 2. Majority ( $\mathrm{n}=185 ; 62 \%$ ) of the participants were screened in one of the two clinics (Figure 2; Table 1). Demographic data for the eligible, ineligible, recruited, and non-recruited participants among those who accepted the study information pack are shown in Table 1.

There were no adverse events during any of the FFMOTs. Ultimately, 25 (86\%) participants completed both questionnaires, representing the number retained throughout the study. Seven of the 25 required follow-up by telephone to complete missing data on the questionnaires.

Table 2 shows the CHAMPS and post-intervention questionnaire outcomes.

\section{Focus groups with older adults}

Twenty-five of the 29 consented participants were invited to attend one of three 60-90 minute focus groups at the same physiotherapy clinic where their FFMOT took place. Four participants were not invited to attend because their FFMOTs took place after the final focus group date had been set. Eight participants did not attend due to health problems $(\mathrm{n}=2)$, unavailability on the day of the focus group ( $n=3)$, holidays $(n=1)$, or unknown reasons $(\mathrm{n}=2)$. In total, 17 participants (median age 70, IQR $67-78 ; 76 \%$ female) attended the first $(n=5)$, second $(n=7)$, or third $(n=5)$ focus group between 2 and 8 weeks after they attended their FFMOT session. Quotes as exemplars of the categories from the main themes of the focus groups are provided in the supplementary material that can be found here: https://edshare.gcu.ac.uk/3386/.

\section{Reasons for undergoing the FFMOT}

There were four main categories resulting from this theme in the focus groups:

- Additional exercises to aid recovery. It is of note that a few participants expected to receive more (or other) 


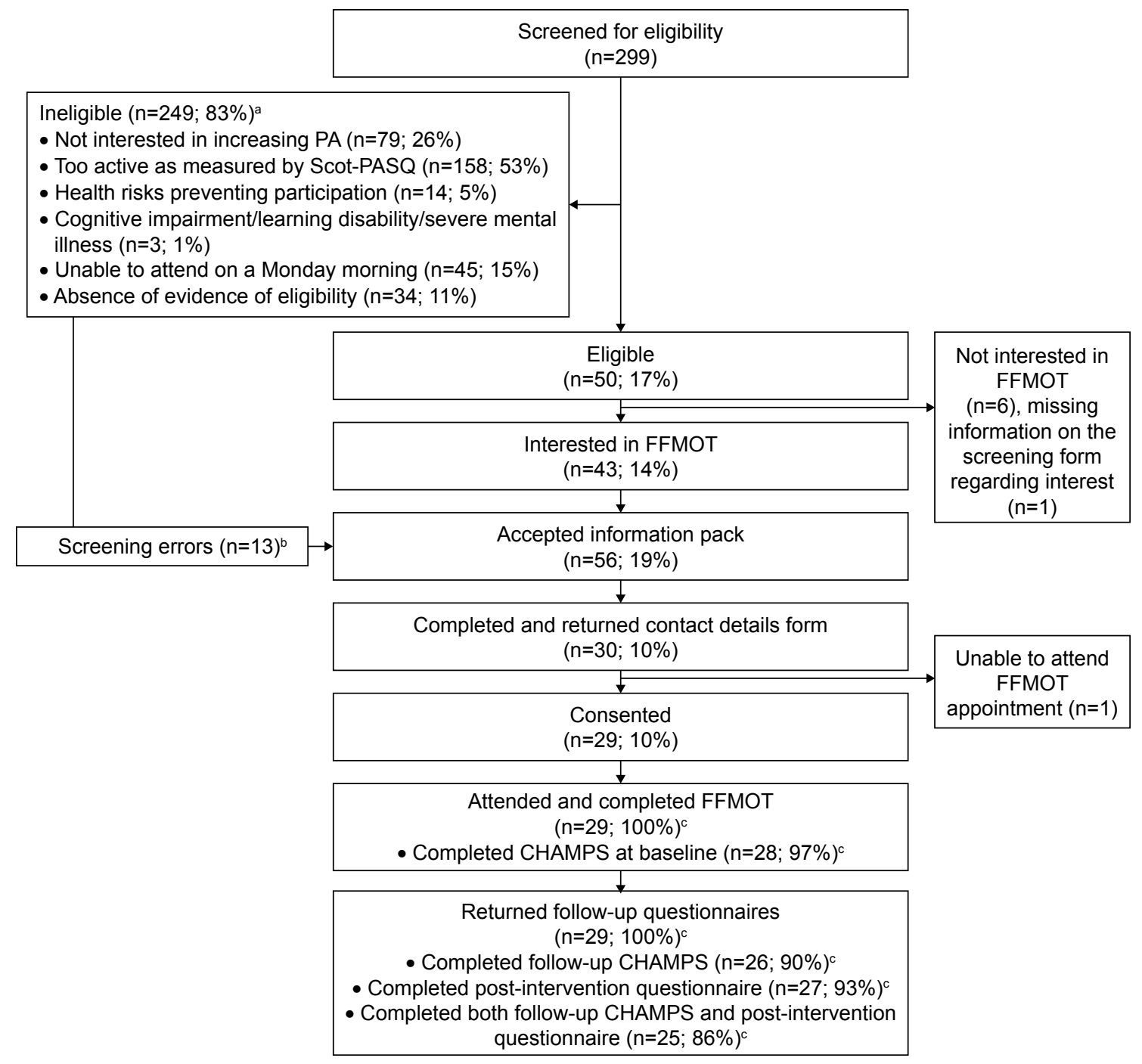

Figure 2 Flow of participants through each stage of the trial from initial screening by PTs to 12-week follow-up.

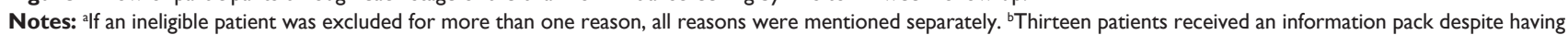
insufficient screening information completed to confirm eligibility $(n=8)$ or not meeting the inclusion criteria $(n=5)$. Of the latter five, one patient was identified as having a health risk precluding exercise (even though this patient was doing 150 minutes of physical activity per week, they chose not to participate after all). 'Denominator used for percentages is the total number consented $(n=29)$.

Abbreviations: CHAMPS, Community Healthy Activities Model Program for Seniors; FFMOT, Functional Fitness MOT; PA, physical activity; PT, physiotherapist; ScotPASQ, Scottish Physical Activity Screening Question.

Table I Demographic characteristics of eligible and ineligible patients among those screened, and of recruited and non-recruited participants among those who accepted the study information pack

\begin{tabular}{|c|c|c|c|c|}
\hline \multirow[t]{2}{*}{ Characteristic } & \multicolumn{2}{|l|}{ Screened $(n=299)$} & \multicolumn{2}{|c|}{ Accepted information pack $(n=56)$} \\
\hline & Eligible $(n=50)$ & Ineligible ( $n=249)$ & Recruited (n=29) & Not recruited $(n=27)$ \\
\hline Age (years), median (IQR) & 7I (67-79) & $69(64-76)$ & $70(67-78)$ & $70(68-75)$ \\
\hline Sex, no of females (\%) & $29(58)$ & $148(60)$ & $21(72)$ & $13(48)$ \\
\hline SIMD 2012 rank, ${ }^{a}$ median (IQR) & $4,998(3,585-6,137)$ & $5,027(3,162-6,194)$ & $5,649(3,559-6,307)$ & $4,998(3,844-6,200)$ \\
\hline Distance from home to clinic (miles), median (IQR) & & & $2.5(1.5-3.7)$ & $3.3(2.6-4.1)$ \\
\hline \multicolumn{5}{|l|}{ Centers } \\
\hline Clinic I, n (\%) & $21(42)$ & $93(37)$ & $16(6 \mathrm{I})$ & $10(39)$ \\
\hline Clinic 2, n (\%) & $29(58)$ & $156(63)$ & $13(43)$ & $17(57)$ \\
\hline
\end{tabular}

Note: aSIMD 2012 divides Scotland into 6,505 datazones, with each datazone being ranked according to an index of multiple deprivation with rank I being the most deprived and rank 6,505 the least deprived.

Abbreviation: SIMD, Scottish Index of Multiple Deprivation. 
Table 2 CHAMPS and post-intervention questionnaire outcomes

\begin{tabular}{|c|c|c|}
\hline Questionnaire outcome & Baseline & I 2 weeks \\
\hline \multicolumn{3}{|l|}{ CHAMPS, median (IQR) } \\
\hline All physical activities & $18(11-28)^{a}$ & $20(1 \mathrm{I}-27)^{\mathrm{b}}$ \\
\hline Moderate-intensity physical activities & $5(2-7)^{\mathrm{a}}$ & $3(2-8)^{b}$ \\
\hline \multicolumn{3}{|l|}{ Post-intervention questionnaire, ${ }^{c}$ yes, $\mathrm{n}(\%)$} \\
\hline $\begin{array}{l}\text { Did attending the FFMOT assist you in identifying local physical activity opportunities or home exercise } \\
\text { opportunities of interest to you? }\end{array}$ & & $21(78)$ \\
\hline Since attending the FFMOT, have you attended any local organized physical activity sessions for the first time? & & $7(25)$ \\
\hline Since attending the FFMOT, do you do more exercise at home or on your own than you did before the FFMOT? & & $13(48)$ \\
\hline Since attending the FFMOT have you taken up any physical activities that you had previously done in the past? & & $7(25)$ \\
\hline $\begin{array}{l}\text { If you did take up any additional physical activity opportunities since attending the FFMOT, are you still participating } \\
\text { in these? }\end{array}$ & & $9(82)^{d}$ \\
\hline
\end{tabular}

Notes: ${ }^{a} n=28 .{ }^{b} n=26 .{ }^{c} n=27 .{ }^{d} n=11$.

Abbreviations: CHAMPS, Community Healthy Activities Model Program for Seniors; FFMOT, Functional Fitness MOT.

exercises in light of the treatment for the specific condition they were attending the clinic for, or for their general recovery.

- PTs recommendation. Some attended the FFMOT purely as they felt this was recommended by the screening PT.

- Curiosity. Others attended out of curiosity and wanted to know more.

- Personalized fitness advice. There was an expectation that they would receive personalized advice on PA to become more active.

\section{Reasons for not being physically active}

There were four main categories within this theme in the focus groups:

- Health related issues. Most participants indicated that they were not physically active because of injuries, pain, or tiredness.

- Perception that they were active enough. There were several participants who perceived themselves as being physically active enough, and as a few people erroneously received an invite to the FFMOT despite reporting being regularly active (using Scot-PASQ), this may be a valid perception.

- Lack of time or other commitments. A few participants mentioned a lack of time or having other commitments that meant they could not commit to regular activity.

- Psychological reasons. A few participants were conscious of the fact that they were not being physically active enough but had not acted on it because they were unmotivated or they feared that they might hold other people back in group activities (not fit enough compared to others).

\section{Changes in perception of, or views on, PA}

Three main categories were identified based on the discussion on this theme. One category was related to no change in the perception or views and the other two on positive changes in perception or views.

- No change. Two codes were combined in this category. The majority of participants experienced no changes in their perception of, or views on, PA because they already perceived themselves as being physically active or they did not feel they learned anything new.

- Change in the understanding of the need for selfmanagement. Two codes were combined in this category. Some participants felt the FFMOT positively influenced their awareness that they were not doing enough and/or it was their own responsibility to be (come) physically active in order to age successfully.

- Change in the awareness of the importance of balance and the need for strength to perform functional activities. Several participants noted their new knowledge around the importance of balance, ways to improve balance, and the need of good strength for certain functional activities.

\section{Changes in awareness of local opportunities to become more physically active}

The majority of participants indicated that the FFMOT had increased their awareness of local opportunities to become more physically active, and some of them found it quite useful to have the information about the local opportunities. However, although they expressed their intention to take up new opportunities, most of them had not yet done so. Four codes appeared from the transcripts from those who had felt their awareness had improved about local opportunities, but 
they had not yet taken up these opportunities because 1) the venue/activity was too far away, 2) the timing of the activities interfered with other commitments, 3) they were unsure of the appropriateness of the activities for them, or 4) they found the information useful, but had just not got around to doing anything about it yet. From those who felt there was no change to their awareness, there were two main reasons ("codes") given: 1) they already knew of the opportunities or 2) they were only interested in doing one activity and so did not look into other activities.

\section{Changes in PA behavior}

Three main codes were merged to form the category of the FFMOT stimulating changes in their PA behavior. Participants reported that they 1) used the home exercise booklet, 2) interrupted sitting more, and 3) had a wider change of health behaviors such as changing their diet. A large number of participants said they used the home exercise booklet after the FFMOT. Specifically, some incorporated exercises into their activities of daily life. Many spoke about interrupting their sitting more during the day. Attendance at the FFMOT motivated one participant to a more general change in health behavior (diet) concerning their weight, perhaps as this was recorded as part of the FFMOT. For some participants, attendance at the FFMOT did not change their PA behavior. These participants gave the same reason(s) as they gave regarding their PA levels prior to the FFMOT (ie, perceived themselves as active already, health issues such as pain, lack of time, or having other commitments).

\section{Appeal of the FFMOT; likes and dislikes}

Participants were asked what they liked or did not like about the FFMOT, what it felt like undergoing the FFMOT, and what it felt like to receive the results of the tests. As their responses to these questions had a lot of overlap, the codes were merged to form categories within the overarching themes of likes or dislikes.

The majority of the participants liked, and expressed positive feelings about, the FFMOT. The codes that were merged to form this category included 1) the one-to-one personal attention, 2) appreciation for the personally tailored advice, and 3) the comparison of their test results to their peers. Some particularly mentioned that they liked specific functional tests, either because they were relevant to them or they raised awareness of how well or how poorly they performed on that component of fitness. One participant was neutral (did not like or dislike anything) about the FFMOT. Others expressed dislike about some components of the FFMOT, including categories covering their dislike of certain tests if they either could not do these well (disappointment/embarrassment) or if they felt they were not challenging enough for them, feelings of the FFMOT being too superficial for them. One person said the FFMOT just made them feel lazy.

\section{Appeal of the FFMOT; recommend it to others?}

The majority of participants would recommend the FFMOT to others for various reasons. Mostly, they felt it was useful for people to know what they could or could not do well, and what to work on to maintain independence in the future. At this point, there was also discussion about the fact that the FFMOT should be offered to those not attending physiotherapy, but who are just generally inactive. There were no participants who expressed the view that they would not recommend the FFMOT to others, although one participant felt neutral about it. Interestingly, even those who felt they were already active enough would have recommended it to others.

\section{Views on location and timing of the FFMOT}

About half of all participants said that the FFMOT should be done within the NHS because they believed that the NHS personnel were the experts and their venues would be best equipped for it. However, many also said it could be run under a community service because that would be closer for, and more accessible to most people. Overall, participants tended to prefer late morning or early afternoon sessions.

\section{Appeal of the FFMOT; recommendations for improvement}

The main recommendation from participants to improve the FFMOT was related to tailoring the FFMOT test set to the individual. For example, some participants would have liked a test for cardiovascular stamina and more challenging or relevant tests for specific activities (eg, stair climbing, stooping, standing up from the floor). Another important recommendation was to repeat the FFMOT after a set amount of time as a means to motivate people to change their behavior. One participant felt there should be a stronger emphasis on obesity and weight. A few participants suggested to merge it with a wider health check, such as having blood pressure monitored by the general practitioner.

Finally, during the focus groups, participants were asked a few questions about their views on the study procedures 
(such as concerns about taking part, clarity of the participant information sheet, experiences with completing the consent procedure and CHAMPS questionnaires). On the whole, the participants had no particular concerns prior to participation, and they found the information sheets and consent procedures clear.

The study period included the Christmas holiday, and some participants remarked that they were less active than usual due to this. As such, some participants were cognisant of the limitations of the CHAMPS questionnaire in accurately recording their PA behavior. This information will be taken into consideration for a future study.

\section{Staff interviews with PTs and Tls}

The four participating PTs were female, aged between 33 and 54 with experience ranging from 10 to 33 years across a range of conditions. One of the PTs was the service lead and two had previous research experience. One TI (female, age 46) had 18 years of experience in working in outpatient MSK patients. She had a Scottish Vocational Qualification level 3 in Diagnostic and Therapeutic support and a Higher National Certificate in Physiotherapy. The other TI (female, age 56) was a qualified exercise practitioner with over 30 years of experience in working with older people in different exercise settings. Both TIs were employees from the participating PT services. They were asked about perceived benefits of the FFMOT, things they would like to improve (did not like about it), where it should be implemented and by whom (roles), and what barriers were there to run the FFMOT in an outpatient setting. Quotes as exemplars of the categories from the main themes of the staff interviews are also provided in the Supplementary material that can be found here: https://edshare.gcu.ac.uk/3386/.

\section{Perceived benefits of the FFMOT}

The PTs perceived the FFMOT to be an easy, quick, and motivating means of highlighting to people what the components of fitness are, how their fitness compares to their peers, and in what components of fitness they scored below average for their age group. Some mentioned the personal touch of the FFMOT as a specific benefit, as well as its perceived ability to motivate people to work on their personal (aspects of) fitness, especially as a result of comparison to normative scores. The TIs believed that the FFMOT was educating and encouraging for the participants, it drew on the participants' competitiveness, it had increased the participants' awareness of the components of fitness, and that the tests connected well to the participants' activities of daily life.

\section{Things to improve the FFMOT}

One PT specifically expressed the concern that the normative data used in the FFMOT would not be representative for people with other (than MSK) conditions and also expressed doubts about the overall validity of the tests. It was also feared that the test results might demoralize those people who scored poorly. The PTs perceived space constraints (especially when using the 6-minute walk test), time constraints by the staff (filling in yet more forms), and accessibility of the venue to be the main barriers to delivering the FFMOT. The TIs would have preferred to have more information within the FFMOT on diet and obesity and thought perhaps there was the potential for information overload on the amount of activity choices offered.

There was some disagreement on the use of the 6-minute walk test, which had been removed from the battery of tests for this setting because of space and time constraints. The PTs tended to think it would have been good to include it if the space allowed. The TIs seemed to be in doubt whether the 6-minute walk test should be a part of the FFMOT, worrying mostly about people's ability to walk for 6 minutes.

\section{Roles}

PTs were of the opinion that the TIs were in a good position to provide the FFMOTs, provided they had received the appropriate training and had back-up support from a PT. They also believed that the FFMOTs could potentially be delivered outside a PT service (eg, in the community delivered by the third sector) or even in people's own homes by TIs without supervision, or by someone else trained in having the PA discussion and having the right skill mix. In contrast, both TIs felt that they might not have the appropriate level of knowledge and skills to prescribe exercises and have a discussion after having performed the FFMOT tests. One felt that if the TIs had a fitness qualification, they would be better placed to have the discussion.

\section{Location}

Due to the current financial pressures within the NHS, and because it should be feasible in terms of caseload and space requirements, the main perception of the staff members was that the FFMOT could potentially be organized outside of a PT service, by third sector (voluntary) organizations, or in a local sports center or a leisure center. One PT felt that these might actually be more appropriate places for running it. Although the TIs felt that the FFMOT could be carried out in a variety of other settings so as to demedicalize the tests, they felt it might be better to do it in a health clinic setting because of issues related to safety, privacy, and accessibility. 


\section{Barriers and enablers for implementation in an MSK service}

All PTs expressed their doubts about whether it would actually be feasible to deliver the FFMOT within the NHS. The main categories of the codes extracted for barriers were the current lack of evidence base for the effectiveness of the FFMOT, space requirements, and financial restraints and governance issues in the current NHS environment. The financial restraints were expected to be the main barriers in terms of the resources (staff time, staff training, staff roles) needed to efficiently deliver the FFMOT.

Two categories emerged as enablers: continuous professional development and having something different to do within the PT/TI role. One of the PTs also experienced a "positive buzz" in the practice when the FFMOTs were going on and received some positive feedback from patients who had participated in it.

\section{Discussion}

The FFMOT is a new approach that aims to raise awareness of the importance of components of fitness such as strength, balance, and flexibility among the population aged over 60 , and highlight the benefits of PA, engage older people in health behavior change discussions, and direct them to appropriate local activity resources. It has received considerable interest, with over 600 health and fitness professionals trained, and organizations have reported on its anecdotal benefits. ${ }^{12}$ However, to date, there has been no robust data on its acceptability or its effectiveness. This study aimed to explore the acceptability and feasibility of running FFMOTs within the physiotherapy outpatient environment and to gather necessary data to inform the feasibility of conducting a definitive study of its effectiveness.

\section{Acceptability/appeal of the FFMOT}

Ten percent of all screened and $54 \%$ of those invited to take part underwent the FFMOT. It is likely that this latter group of people were in a stage of contemplation to change PA behavior. This percentage differs considerably from a group exercise intervention lasting 6 months organized through primary care, where only $13 \%$ of those invited took part. ${ }^{10}$ This will have been influenced by the FFMOT being a single-point rather than a long-term intervention. Over half accepting the offer to attend an FFMOT is relatively high considering that these participants were attending MSK outpatient clinics and were currently undergoing rehabilitation for injuries/pain. A significant number of them were not offered the FFMOT as, according to the Scot-PASQ, ${ }^{17} 53 \%$ were already meeting the PA guidelines. This is higher than the recently published PA data in Scotland, which suggests that $44 \%$ of men and $34 \%$ of women aged $65-74$ years and $32 \%$ of men and $19 \%$ of women aged $75+$ years meet the MVPA guideline. ${ }^{28}$ Ineligibility on PA grounds was the principal cause of a low recruitment rate of $10 \%$. However, in terms of appeal, 56 patients accepted an information pack, of whom 30 consented to take part, suggesting that $54 \%$ found the idea of an FFMOT appealing.

People living in low socioeconomic areas and the older/ frailer are less likely to engage with PA. ${ }^{29}$ The screening data showed no meaningful differences in the distance from home to clinic or the index of multiple deprivation between those who were ultimately recruited and those who were not recruited. This suggests that there were no major inequalities in the uptake of the FFMOT. It is to be noted that more women than men were screened and recruited during the study, which may reflect a sex difference in attitudes toward physical activities.

\section{Reports of behavior change}

The post-intervention questionnaire suggested that $78 \%$ of participants felt the FFMOT helped them identify new PA opportunities, but only $25 \%$ started a new organized PA opportunity (or one they had done in the past). However, $48 \%$ said they started doing some exercises at home (from home exercise booklets). Pleasingly, $82 \%$ were still participating in the new PA opportunity at follow-up 12 weeks later. This change in reported behavior in the post-intervention questionnaire was not seen in the CHAMPS data, so many of these activities were either in replacement of something else they used to do or CHAMPS is not sensitive enough to detect these new strength/balance or "break up prolonged sitting" activities. Reasons given for not being physically active were the same as those seen in previous research with older adults, that they had time or other commitment issues, ${ }^{30}$ but health issues seemed to play a larger role, which is perhaps to be expected as this study was recruiting from outpatient MSK clinics.

\section{Views about the effectiveness of the FFMOT from participants and therapy health professionals}

Reasons given by participants for attending the FFMOT were varied. Some said receiving a recommendation from the screening PT encouraged them to attend, which has been seen in previous work where a significant other or health professional increased the likelihood of behavior change. ${ }^{30}$ 
Others confused the aim of the FFMOT with a perceived ability to receive more rehabilitation and exercises for the condition they were attending the clinic for. Some attended as they understood that the FFMOT would offer a more personalized and tailored advice opportunity, and some did not know what to expect but were curious, so came along anyway. The PTs and TIs felt that the FFMOT was quick and gave a chance for personalized feedback that patients could reflect on. They felt it would not only increase awareness of the different components of fitness, but also raise awareness on which particular components each patient should work to improve. They liked the element of competition with the use of normative data for comparison and the "personal touch" that the FFMOT allowed with each patient.

Most participants liked taking part in the FFMOT and cited personalized attention, learning more from specific functional tests about their abilities and comparisons with their peers. These are key components suggested in behavior change interventions, ${ }^{11}$ and it appears they were liked by participants. Those who expressed dislike tended to be unhappy, disappointed, or even embarrassed with particular tests they were not very good at. One felt it was too superficial and one left feeling that they were lazy. Although the TIs made sure they focused on the positives rather than the negatives when giving personal feedback on the test results, for some participants, the comparison with sex- and age-referenced norms led to feelings of disappointment and annoyance. This has to be considered in future discussions with the participants following the FFMOT.

Most participants felt that their awareness of the need for PA had not changed. Many felt they were already active enough and knew the reasons for being active. However, some discussed their new awareness of the need to selfmanage their conditions and help themselves by being more active. There was also a change in perception about the value and importance of good balance and the need for strength to be able to perform certain functional activities. The PTs and TIs also felt that the FFMOT had helped people understand the value of strength and balance work, and some participants had made use of the home exercises incorporating these components of fitness. Some had fitted the exercises in activities of daily living, as suggested by the TIs, which would likely have increased the sustainability of them doing these extra activities. According to recent PA data, only $14 \%$ of men and $16 \%$ of women aged $65-74$ years and $12 \%$ of men and $5 \%$ of women aged $75+$ years meet the strength PA guideline..$^{7,28}$ It seems that the FFMOT has raised awareness of the balance and strength guidelines and the need for strength and balance activities. Interestingly, many participants also voiced incorporating interruptions in prolonged sitting into their daily lives, suggesting that the FFMOT had also raised awareness of the sedentary behavior reduction guideline. It has to be considered that these components of fitness and the simple activities that could be incorporated in order to help meet these guidelines may have had particular attention given by the TIs during the FFMOT, or may have been considered "easier" to incorporate by the participants than finding, traveling, and regularly attending organized activities.

The majority of participants also indicated that the FFMOT increased their awareness of local opportunities to become more physically active and they had considered taking up new opportunities. However, despite good intentions, most of them had not acted on it. There were a variety of reasons given for this, including distance to these opportunities, timings of activities not being helpful with other commitments, or current/ongoing levels of pain/health issues (that they were attending the clinic for). This may be due to the sampling strategy and possibly may have been different if people were not recruited from the outpatient setting. However, it also suggests that many of these individuals were not yet ready to make a change in their PA behavior or needed more motivation to make more significant changes to behavior. This is borne out by participants' recommendations to include a follow-up visit for future FFMOTs to act as a motivator to actually change behavior.

A number of participants voiced that they would have liked a stamina or endurance test within the FFMOT. The original FFMOT test battery does include an endurance assessment, but this was removed from the feasibility study because of space (mostly) and the additional time (6-10 minutes) to administer and discuss the test. It appears that perhaps it should be an optional test for those who want to know more about their stamina. Interestingly the PTs also suggested that if the FFMOT were to be held outside the clinic environment, it might be good to include the 6-minute walk test. The TIs were, however, concerned about the ability of patients within the outpatient setting to complete 6 minutes of walking without difficulty or risk, as some older adults in this setting are more likely to be very deconditioned. The TIs were also concerned about possible demoralization associated with inability to complete the test. This was also a concern from one PT who worried that some older people may score poorly in all tests and be demoralized as a result. In contrast, some participants felt they wanted harder tests or more variety in tests depending on an individual's ability and interests, and some wanted more emphasis on weight 
management or felt the FFMOT would fit better within a wider health check like a health fair or alongside blood pressure monitoring. The TIs agreed that the FFMOT would benefit from a greater emphasis on weight and diet.

The PTs were concerned with the representativeness of the tests (eg, one test of static balance only) and just how accurate they were in people with different conditions (eg, in patients with multiple sclerosis) for whom there is no normative data available. Future research is needed to fill this gap.

During the focus groups, participants were asked if they would recommend attending an FFMOT to other older people, and all (except one who had neutral views on this) would recommend it to others. Although many thought they were active enough already and did not need the FFMOT themselves (or did not agree with particular tests), they did feel it would be useful for "others". This is mirrored in literature about sedentary behavior and falls prevention interventions, where many older people thought they were active enough for health and that falls prevention was a great idea for others but not necessarily for themselves. ${ }^{31,32}$

\section{Views about who should run FFMOTs and where they should be offered}

Both participants and the health professionals felt the health clinic setting was appropriate, and that health professionals had the skills to run the FFMOT. However, most also felt it would be acceptable to run it outside the health setting in community venues so long as the person delivering the FFMOT had been trained and had relevant skills and expertise. Interestingly, the TIs were unsure they had the knowledge and skills necessary for prescribing exercises. They also felt that a greater knowledge of fitness and exercise was necessary for the discussion to be effective. Participants felt that midmorning and early afternoon were the best times to run FFMOTs for older people.

\section{Barriers and enablers for future implementation within an MSK outpatient service}

PTs and TIs both cited the potential cost and time of implementing the FFMOT routinely within an MSK service as a barrier. Many feared it would be difficult to incorporate it into the NHS, given the current financial climate. Space was also considered an issue, especially for reinstating the 6-minute walk test. The PTs cited the current lack of evidence on effectiveness as a barrier. Both the TIs and PTs felt that there was an important role for PTs in the FFMOT referral process, but felt it would perhaps be best implemented in a community setting, with the ability of the PTs to refer people to a service outside the clinic environment. This would help demedicalize the FFMOT and reduce the constraints of space, staff time, and finances.

\section{Considerations for a future definitive study}

This study found that there was a notable difference in screening numbers between the two clinics. The initial estimates of the effect sizes of the CHAMPS were also very small (although it was not powered to show change). These two factors will be used to inform recruitment strategies for a definitive study. For example, for a future trial, 1,000 people would need to be screened in order to recruit $100(10 \%)$ participants. We also found that more than half of those screened for eligibility reported being too active. Because it is highly unlikely that people who are attending an MSK clinic are more active than the general population, this finding suggests a reporting inaccuracy. Other explanations could be that the screening PTs did not place enough emphasis on MVPA when administering the screening Scot-PASQ, or that people reported they were active even when they were not, in order to avoid having to have a conversation about PA. This raises issues with using the Scot-PASQ as a screening tool and it suggests that a more valid self-reported PA measure should be used in a future study, or that training is required to ensure the patients understand the difference between moderate-intensity activity and light PA. Issues with difficulties in ascertaining accurate baseline PA status via self-report have been reported previously. ${ }^{10}$

Based on estimates of the very small effect sizes of the CHAMPS, the sample size needed for a definitive RCT would need to be restrictively large. Although there was an extremely good completion rate for CHAMPS (suggesting it is a good candidate outcome measure for a definitive trial in terms of participant acceptability), our participants voiced concerns about the CHAMPS' ability to objectively capture their PA. Indeed, the CHAMPS is mainly aimed at recording MVPA and does not have specific questions about strength and balance activities or about sitting and sedentary behavior. Using alternative measures of PA, such as the Phone-FITT (where FITT stands for Frequency, Intensity, Time, and Type of activity) or the International Physical Activity Questionnaire, should be considered. As only a quarter of the participants reported taking up new activity opportunities and many still spoke of ongoing pain or discomfort from the issue they had been attending the clinic for, it would be 
worthwhile having a longer-term follow-up so that delayed changes in behavior can be captured. Indeed, many participants spoke of a follow-up FFMOT and how this might be a motivator to do more. For a future study, the location where the FFMOT is offered should also be re-evaluated, as well as who will deliver it. When originally developed, the intention was that the FFMOT should be delivered in shopping centers and health fairs for older people, and be delivered by anyone working with older people following some brief training. However, as allied health professionals are tasked with raising awareness about $\mathrm{PA}$ with their older patients, the clinics specifically wanted to test its feasibility in this setting. Given the perceived barriers to implementation within an MSK setting, and the feedback from participants, it would be worthwhile to compare the uptake and effectiveness of the delivery of the FFMOT in different settings using a variety of FFMOT-trained deliverers. With this design, the appeal to different sexes and people from different socioeconomic backgrounds could be considered. Finally, in a future definitive study, a logic model should be developed that allows a more structured interview schedule to explore differences between those who change their behavior and those who do not, so that a theoretical framework of implementation can be developed.

\section{Strengths and limitations of this study}

The main strength of this study is that it has investigated the views and perceptions of the FFMOT (appeal and potential effectiveness) from both the potential consumers' (older people) and the health care professionals; (PTs, TIs) perspective.

This was a feasibility study, and the main limitation is the potential lack of generalizability. The participants in this study were selected from the caseloads of community PTs working in two physiotherapy clinics for MSK conditions. As such, this sample is not representative of healthy older adults or people with other (eg, neurological) conditions. In fact, in some instances, the participants' conditions for which they were receiving treatment within the MSK clinic prevented them from undertaking more PA. A considerable number of participants were expecting the FFMOT to be a continuation of their physiotherapy treatment, probably because the FFMOT was delivered in the same setting where they received treatment. These participants might not have participated had they been made more consistently aware that the FFMOT was not intended as an extension of their treatment. All these factors may have influenced our results.
There were other limitations, which include errors in screening and the lack of emphasis on structured PA opportunities by the TIs during the conversation that followed the FFMOT tests. For example, during the screening process, 13 participants received an information pack in error. Three of these 13 underwent the FFMOT and participated in the focus groups despite the fact that they should have been excluded due to already achieving the volume of PA as recommended for health. Their participation may have influenced the focus group discussions. The occurrence of screening errors suggests that some PTs were either unclear about the eligibility criteria for the study or found it challenging to check them properly within the context of a busy outpatient service. Both participating TIs reported that during the discussion after the tests, they mainly focused on the home exercises rather than the local opportunities, as they were more familiar with these. This may have affected the motivation of the older people to pursue the organized local opportunities. Finally, although most participants felt positive about receiving their personal FFMOT test results, the question remains whether this was because they scored well or they were relieved to find out that they did not score worse. This distinction may be important to be explored in future research.

\section{Conclusion}

The overall results of this feasibility suggest that the FFMOT is appealing and potentially feasible in the clinical setting. The recruitment and retention data suggest that it should be feasible to conduct a definitive RCT which investigates whether the FFMOT is effective at promoting increased moderate-intensity PA, sitting less, and incorporating strength and balance activities into daily activity routines in this population. However, amendments to some aspects of the screening procedure and the outcome measures are needed.

\section{Acknowledgments}

The authors would like to thank Irene Carter and Alison Hamilton for administering the FFMOTs and all participants for their participation. Andy D Peters was supported by the Edinburgh Clinical Trials Unit. The intellectual property of the FFMOT resides with Glasgow Caledonian University.

\section{Disclosure}

DAS and RL are directors of Later Life Training Ltd, a notfor-profit organization that delivers training on delivery of the FFMOT, motivation to exercise, and exercise delivery for older people. SG is a senior tutor/assessor for Later Life 
Training Ltd. The authors report no other conflicts of interest in this work.

\section{References}

1. Landi F, Abbatecola AM, Provinciali M, et al. Moving against frailty: does physical activity matter? Biogerontology. 2010;11(5):537-545.

2. Department of Health Physical Activity Health Improvement and Protection. Start Active, Stay Active. Report. 2011. Available from: https:// www.gov.uk/government/uploads/system/uploads/attachment_data/ file/216370/dh_128210.pdf. Accessed May 8, 2018.

3. Davis MG, Fox KR, Hillsdon M, Sharp DJ, Coulson JC, Thompson JL. Objectively measured physical activity in a diverse sample of older urban UK adults. Med Sci Sports Exerc. 2011;43(4):647-654.

4. Sun F, Norman IJ, While AE. Physical activity in older people: a systematic review. BMC Public Health. 2013;13:449.

5. Jefferis BJ, Sartini C, Ash S, et al. Trajectories of objectively measured physical activity in free-living older men. Med Sci Sports Exerc. 2015;47(2):343-349.

6. Long-term Conditions Alliance Scotland. PATH to active ageing. Promoting the benefits of physical activity for older people; 2012. Available from: https://agescotland.files.wordpress.com/2013/01/ active_ageing_report_revised-1.pdf. Accessed May 8, 2018.

7. Strain T, Fitzsimons C, Kelly P, Mutrie N. The forgotten guidelines: cross-sectional analysis of participation in muscle strengthening and balance \& co-ordination activities by adults and older adults in Scotland. BMC Public Health. 2016;16(1):1108.

8. Harvey JA, Chastin SF, Skelton DA. How sedentary are older people? a systematic review of the amount of sedentary behavior. J Aging Phys Act. 2015;23(3):471-487.

9. Lowe A, Gee M, Mclean S, Littlewood C, Lindsay C, Everett S. Physical activity promotion in physiotherapy practice: a systematic scoping review of a decade of literature. Br J Sports Med. 2018;52(2): $122-127$.

10. Iliffe S, Kendrick D, Morris R, et al. Promoting physical activity in older people in general practice: ProAct65+cluster randomised controlled trial. Br J Gen Pract. 2015;65(640):e731-e738.

11. Michie S, van Stralen MM, West R. The behaviour change wheel: a new method for characterising and designing behaviour change interventions. Implement Sci. 2011;6:42.

12. BHF National Centre for Physical Activity and Health. Later Life Training, BHF National Centre for Physical Activity and Health. The functional fitness MOT programme impact assessment; 2014. Available from: http://www.laterlifetraining.co.uk/wp-content/ uploads/2014/08/Functional-Fitness-MOT-impact-assessment_GCU_ LLT_BHFNCPAH_2014.pdf. Accessed May 8, 2018.

13. Rikli RE, Jones CJ. Development and validation of criterion-referenced clinically relevant fitness standards for maintaining physical independence in later years. Gerontologist. 2013;53(2):255-267.

14. National Institute for Health and Care Excellence. Behaviour change: general approaches. Manchester; 2007. Available from: https://www. nice.org.uk/guidance/ph6. Accessed September 6, 2018.

15. de Jong LD, Peters A, Hooper J, et al. The functional fitness MOT test battery for older adults: protocol for a mixed-method feasibility study. JMIR Res Protoc. 2016;5(2):e108-e108.
16. Bowen DJ, Kreuter M, Spring B, et al. How we design feasibility studies. Am J Prev Med. 2009;36(5):452-457.

17. NHS Health Scotland. Scottish physical activity screening question (Scot-PASQ). Available from: http://www.paha.org.uk/Resource/ scottish-physical-activity-screening-question-scot-pasq. Accessed May 8, 2018.

18. Rikli RE, Jones CJ. Functional fitness normative scores for communityresiding older adults, ages 60-94. J Aging Phys Act. 1999;7(2): $162-181$.

19. El-Sobkey SB. Normative values for one leg stance balance test in population based sample of community dwelling older people. Middle East J Sci Res. 2011;7:497-503.

20. Later Life Training Home Exercise Booklets. Available from: http:// www.laterlifetraining.co.uk/llt-home-exercise-booklets/. Accessed May 8, 2018.

21. Later Life Training, About Motivate Me. Available from: http://www. laterlifetraining.co.uk/courses/motivate-me/about-motivate-me/. Accessed May 8, 2018.

22. Stewart AL, Mills KM, King AC, Haskell WL, Gillis D, Ritter PL. CHAMPS physical activity questionnaire for older adults: outcomes for interventions. Med Sci Sports Exerc. 2001;33(7):1126-1141.

23. Hekler EB, Buman MP, Haskell WL, et al. Reliability and validity of CHAMPS self-reported sedentary-to-vigorous intensity physical activity in older adults. J Phys Act Health. 2012;9(2):225-236.

24. The Scottish Government. Scottish index of multiple deprivation 2012: a national statistics publication for Scotland; 2012. Available from: http://www.gov.scot/Topics/Statistics/SIMD. Accessed May 8, 2018.

25. Distance between UK Postcodes. Available from: https://www. freemaptools.com/distance-between-uk-postcodes.htm. Accessed May 8, 2018.

26. Krippendorff K. Content Analysis: An Introduction to its Methodology. 2nd ed. Thousand Oaks: SAGE; 2004.

27. Graneheim UH, Lundman B. Qualitative content analysis in nursing research: concepts, procedures and measures to achieve trustworthiness. Nurse Educ Today. 2004;24(2):105-112.

28. Brown L, Campbell-Jack D, Gray L, Hovald P, Kirkpatrick G, Knudsen L. The Scottish Health Survey 2015; 2016. Available from: http://www. webcitation.org/6nWg8ZuyH. Accessed January 15, 2017.

29. Farrell L, Hollingsworth B, Propper C, Shields MA. The socioeconomic gradient in physical inactivity: evidence from one million adults in England. Soc Sci Med. 2014;123:55-63.

30. Finch H. Physical Activity "At Our Age": Qualitative Research among People over the Age of 50. London: Health Education Authority; 1997.

31. Yardley L, Donovan-Hall M, Francis K, Todd C. Older people's views of advice about falls prevention: a qualitative study. Health Educ Res. 2006;21(4):508-517.

32. Haines TP, Day L, Hill KD, Clemson L, Finch C. "Better for others than for me": a belief that should shape our efforts to promote participation in falls prevention strategies. Arch Gerontol Geriatr. 2014;59(1): 136-144.

33. Later Life Training and Glasgow Caledonian University. The Functional Fitness MOT: Course Manual and Resources. Edition 1. Killin: Later Life Training; 2017. 


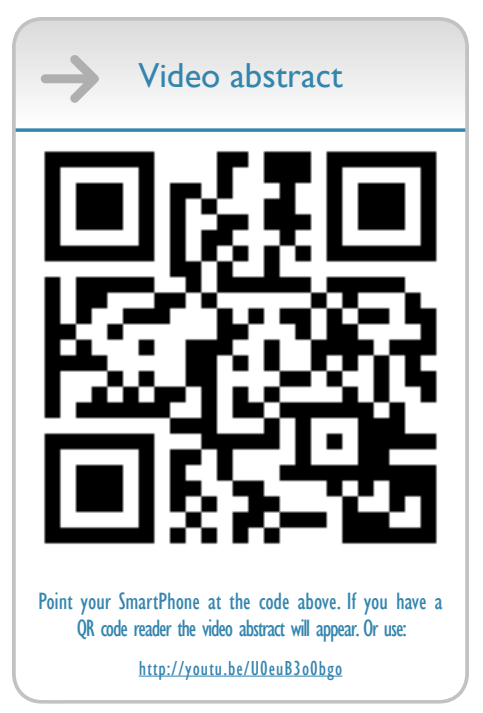

Clinical Interventions in Aging

Dovepress

\section{Publish your work in this journal}

Clinical Interventions in Aging is an international, peer-reviewed journal focusing on evidence-based reports on the value or lack thereof of treatments intended to prevent or delay the onset of maladaptive correlates of aging in human beings. This journal is indexed on PubMed Central, MedLine,
CAS, Scopus and the Elsevier Bibliographic databases. The manuscript management system is completely online and includes a very quick and fair peer-review system, which is all easy to use. Visit http://www.dovepress. com/testimonials.php to read real quotes from published authors. 